Supporting Information

\title{
SARS-CoV-2 Variants are Selecting for Spike Protein Mutations that Increase Protein Stability
}

David Shorthouse, Benjamin A. Hall*

Department of Medical Physics and Biomedical Engineering, University College London,

Gower Street, London, United Kingdom, WC1E 6BT

*Corresponding author Email: B.Hall@ucl.ac.uk 


\section{Experimental procedures:}

\section{$\Delta \Delta \mathrm{G}$ Calculation:}

To study the mutational landscape of the SARS-CoV-2 spike protein from PDBID 6VXX ${ }^{1}$, the structure was initially relaxed and repaired using the RepairPDB command in Fold $x 4^{2}$ as follows:

\$foldx --command=RepairPDB --pdb=6vxx.pdb --ionStrength=0.05 --pH=7 --vdwDesign=2

RepairPDB was repeated on the structure six times to minimize its energy. The relaxed structure was then used to calculate the $\Delta \Delta \mathrm{G}$. PositionScan was run on each residue in the protein structure sequentially using the following command:

\$foldx --command=PositionScan --pdb=6vxx_repaired.pdb --ionStrength=0.05 --pH=7 -vdwDesign=2 --pdbHydrogens=false --positions $=100$

To run PositionScan on the $100^{\text {th }}$ residue. PositionScan mutates a target residue sequentially from wildtype (WT) to each amino acid possibility, calculating the $\Delta \Delta \mathrm{G}$ relative to wildtype each time. The protein backbone is unchanged, but the energy cost or gain from inducing a different side chain is measured. Histidine protonation state is calculated in each case from the input $\mathrm{pH}(7)$ and the surrounding side chains.

\section{Mutations:}

Mutations in SARS-CoV-2 variants were obtained from CoVariants ${ }^{3}$ (https://covariants.org/). 


\section{Expected mutational $\Delta \Delta \mathrm{G}$ :}

To calculate the expected mutational $\Delta \Delta \mathrm{G}$ for a variant (Figure $\mathrm{S} 1$ ), 1,000,000 samples of the same number of mutations in the variant were taken from the structure. For each sample the $\Delta \Delta \mathrm{G}$ was calculated and the median of the distribution taken as the expected value. The value observed for the variant was removed from the expected to generate the $\Delta \Delta \mathrm{G}$ difference.

\section{Mutational $\Delta \Delta \mathrm{G}$ combinations:}

To calculate the $\Delta \Delta \mathrm{G}$ for combinations of mutations in each variant, every possible combination of mutations in each variant was calculated. Each combination was then generated 15 times and average $\Delta \Delta \mathrm{G}$ calculated using the Foldx BuildModel command:

\$foldx --command=BuildModel --pdb=6vxx_repaired.pdb --mutant-file=mutantfile.txt -numberOfRuns $=15$--pH=7 --vdwDesign $=2$--ionStrength $=0.05$

Where mutant-file.txt is a file containing the mutational combination to be modelled separated by a comma. For example, to model mutations L452R, D614G, and D950N in the Delta variant the file would contain:

\section{LA452R,DA614G,DA950N;}

\section{Supplementary Figures:}

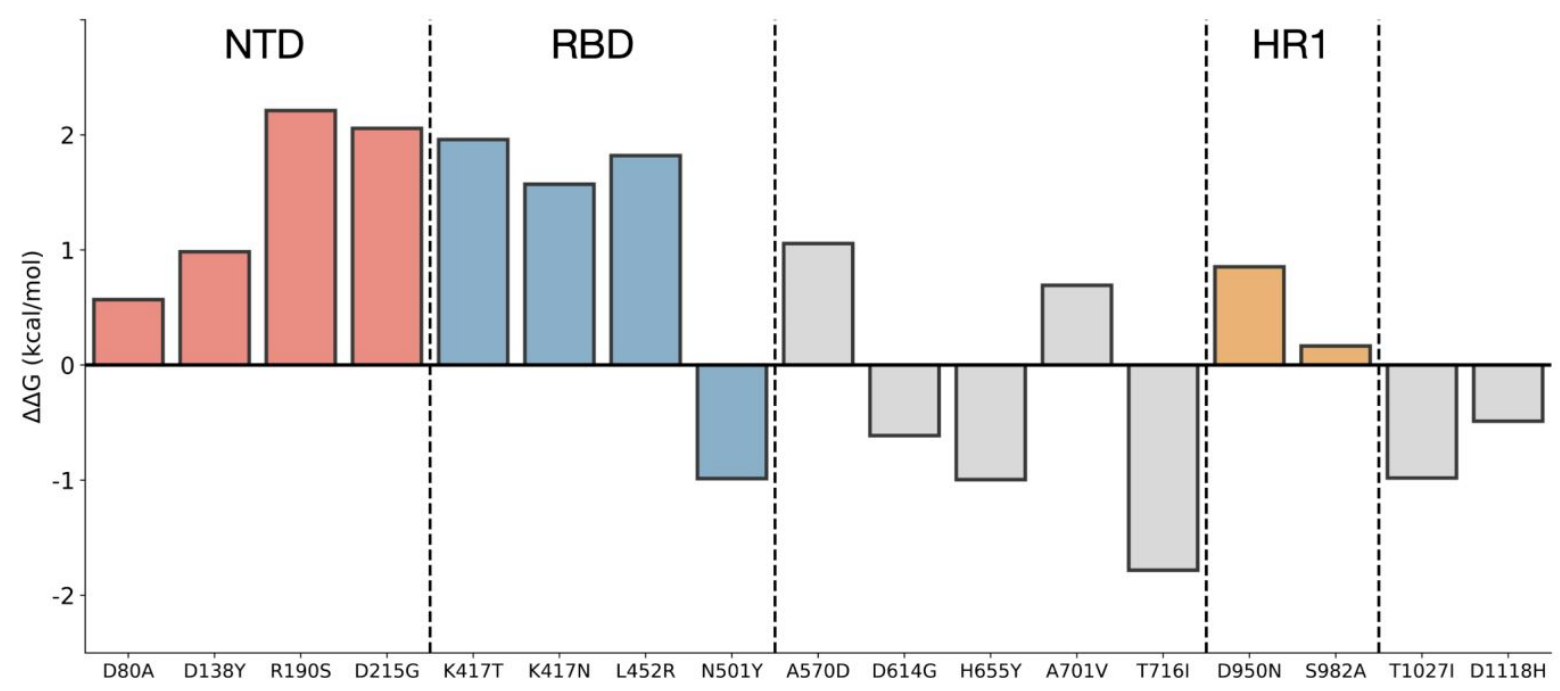


Figure S1: Mutational $\Delta \Delta \mathrm{G}$ for mutations coloured by location in the spike protein. (NTD N-terminal doman, RBD - Receptor Binding Domain, HR1 - Heptapeptide repeat 1).

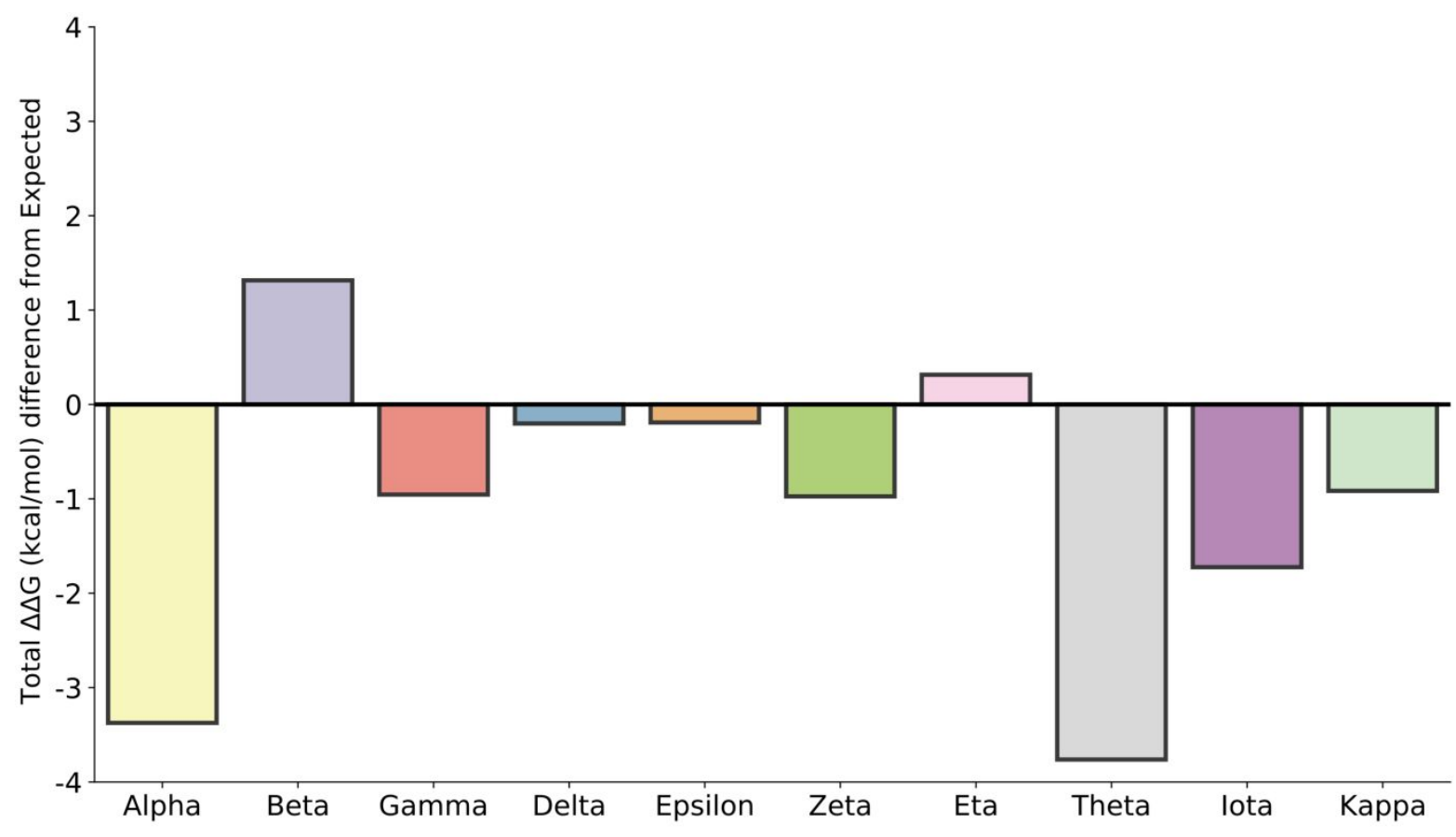

Figure S2: Difference between median expected $\Delta \Delta \mathrm{G}$ for each variant and observed $\Delta \Delta \mathrm{G}$ $(\mathrm{Kcal} / \mathrm{mol})$ 


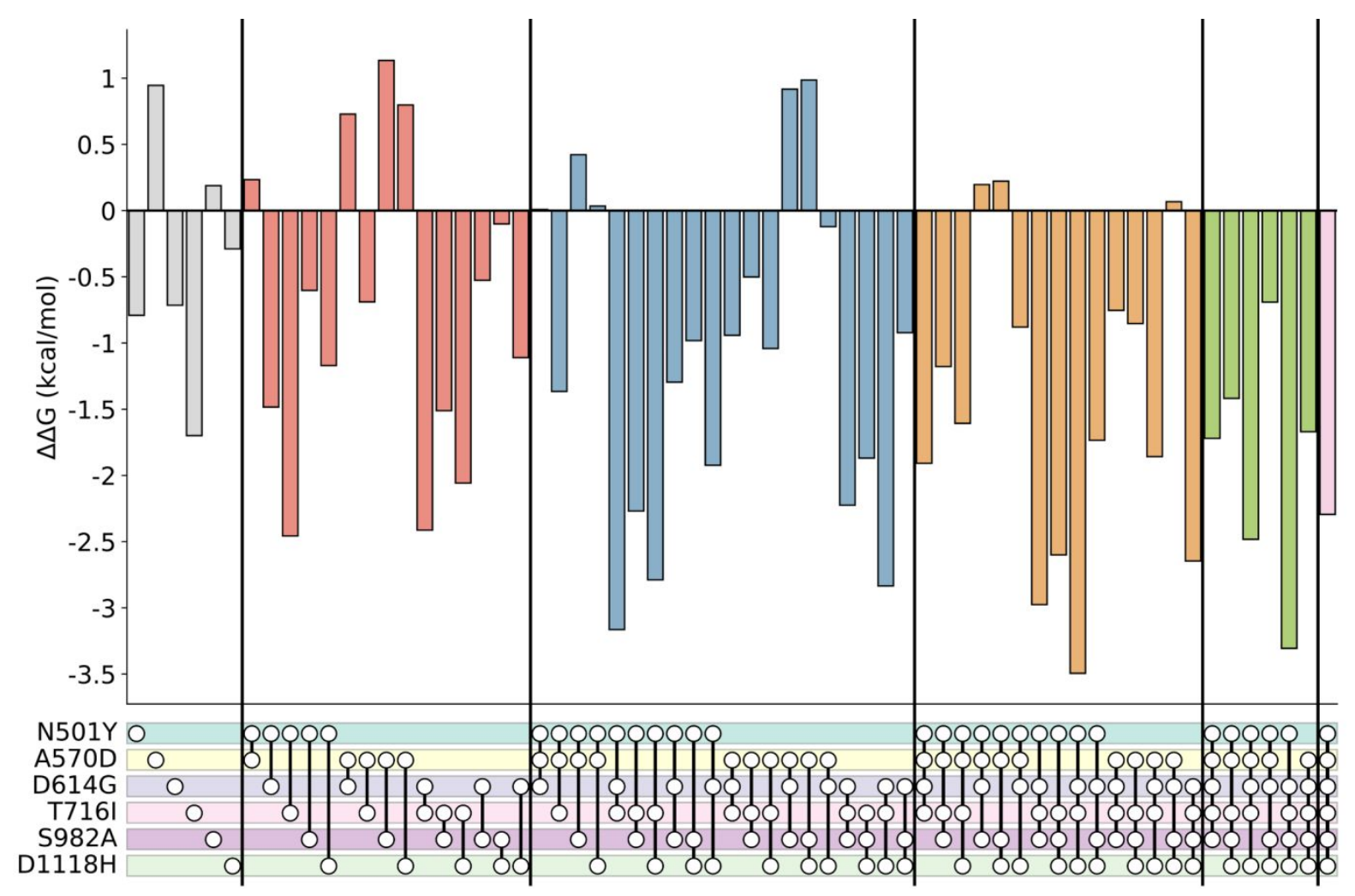

Figure S3: Upset plot for mutation combinations in SARS-CoV-2 Alpha variant.

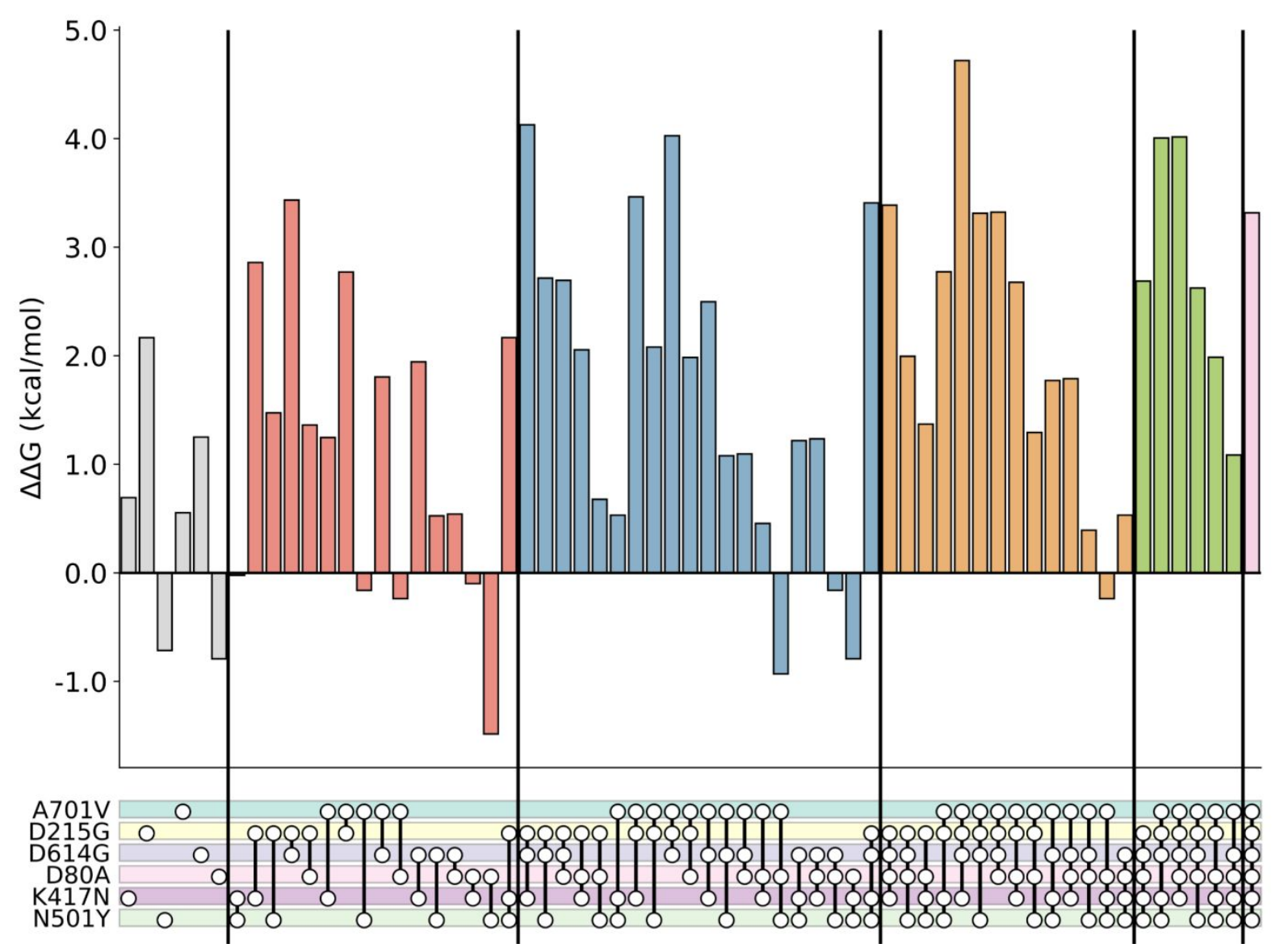

Figure S4: Upset plot for mutation combinations in SARS-CoV-2 Beta variant 


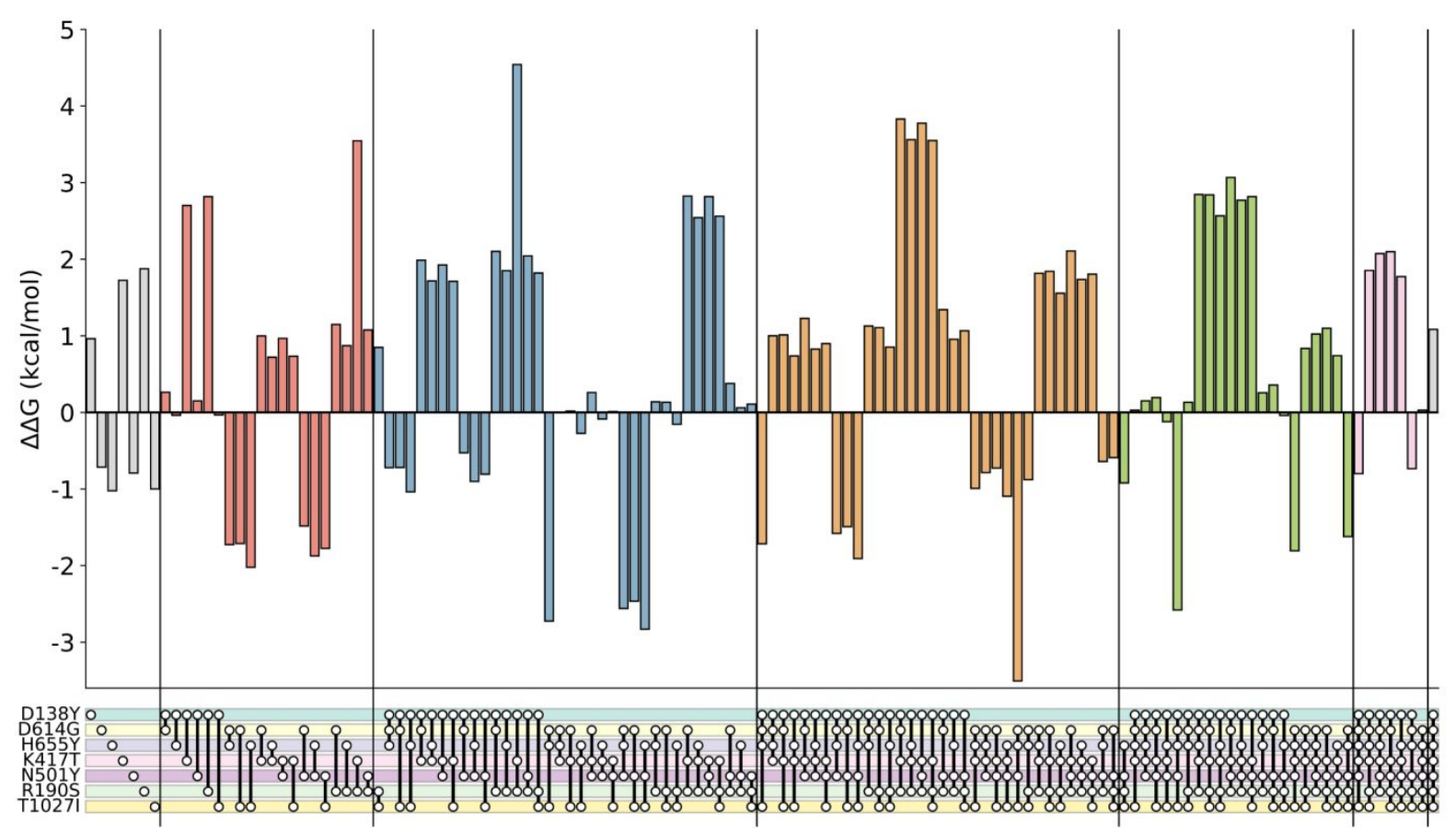

Figure S5: Upset plot for mutation combinations in SARS-CoV-2 Gamma variant.

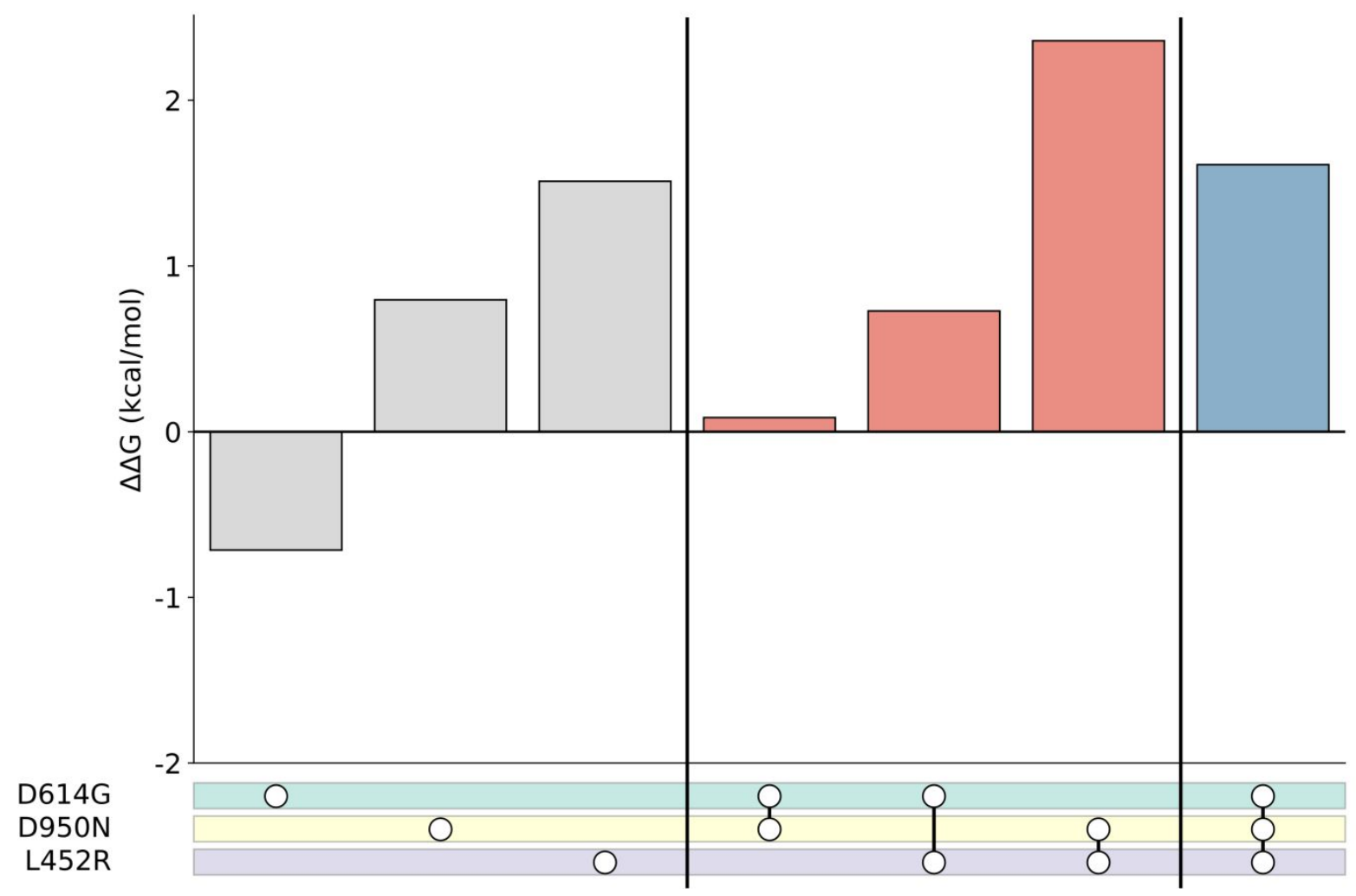


Figure S6: Upset plot for mutation combinations in SARS-CoV-2 Delta variant.

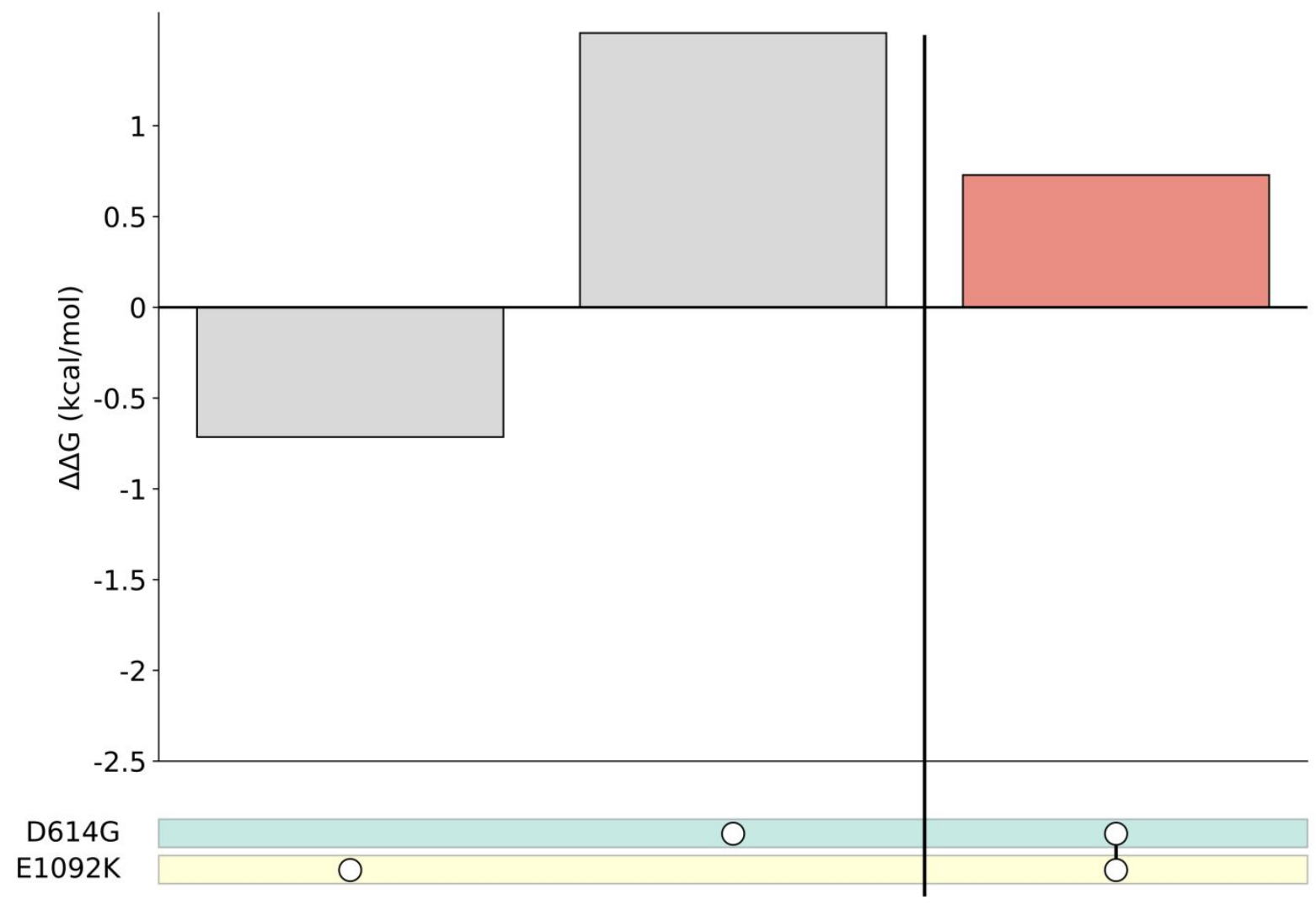

Figure S7: Upset plot for mutation combinations in SARS-CoV-2 Epsilon variant.

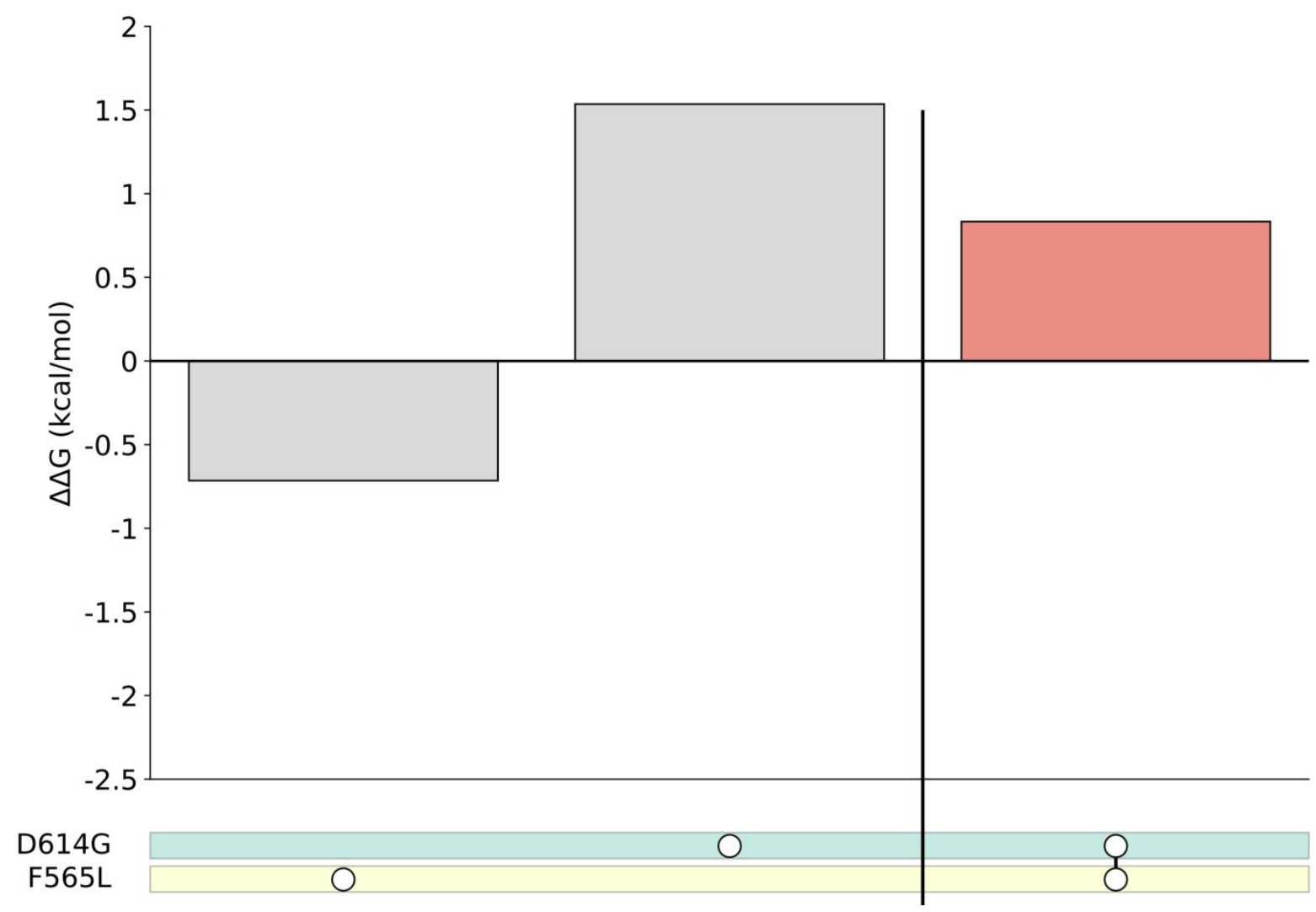


Figure S8: Upset plot for mutation combinations in SARS-CoV-2 Zeta variant.

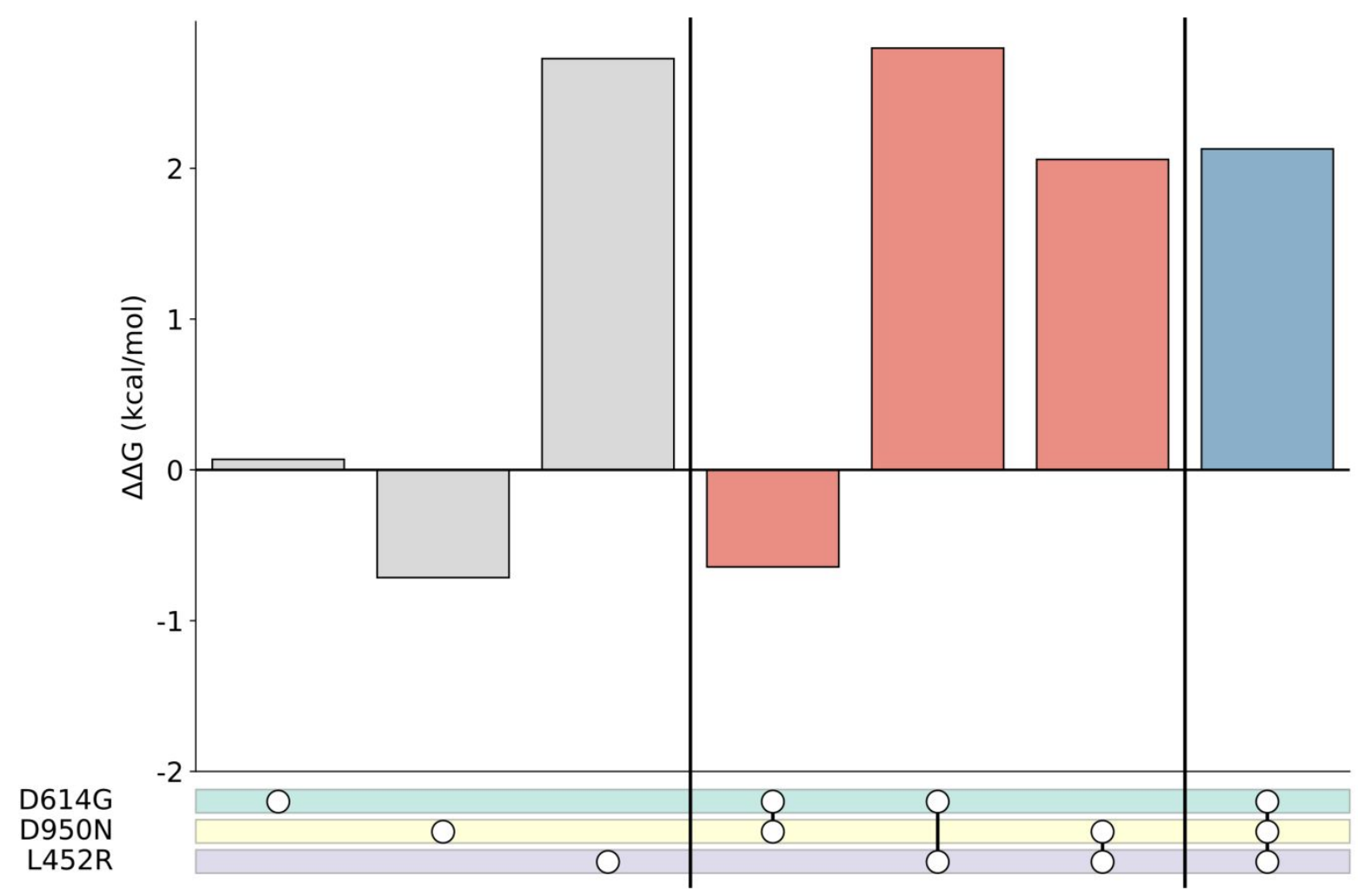

Figure S9: Upset plot for mutation combinations in SARS-CoV-2 Eta variant. 


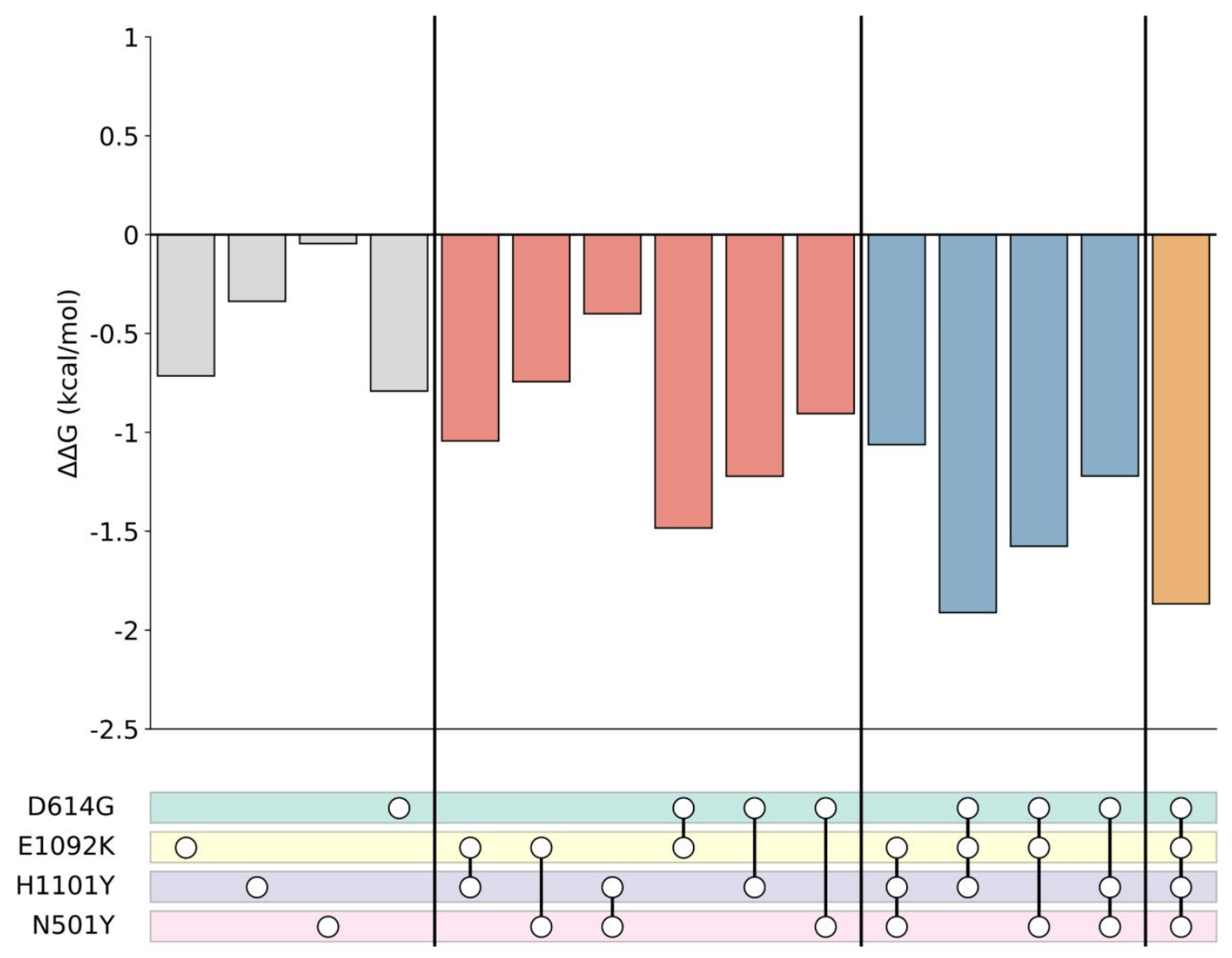

Figure S10: Upset plot for mutation combinations in SARS-CoV-2 Theta variant.

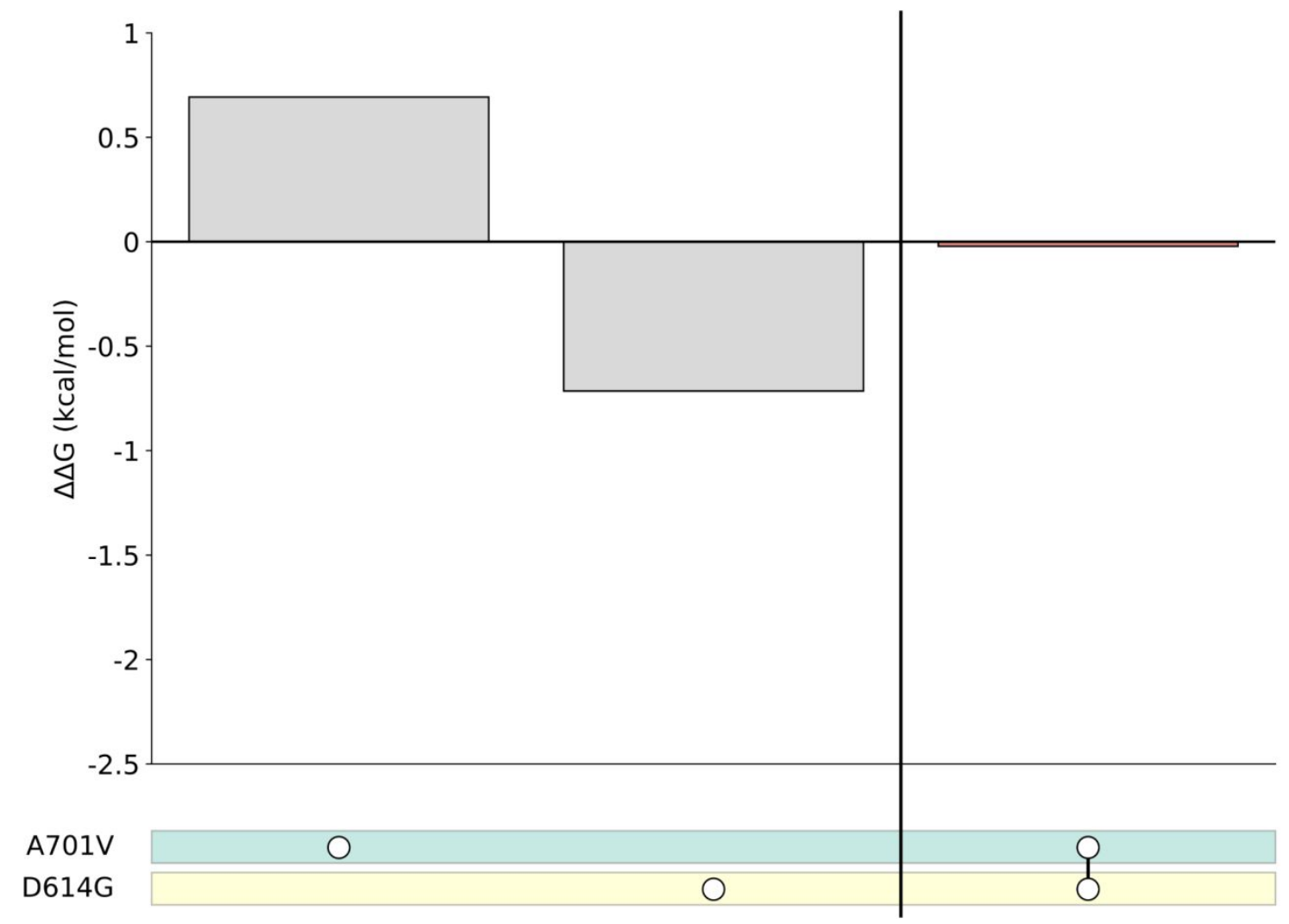


Figure S11: Upset plot for mutation combinations in SARS-CoV-2 Iota variant.

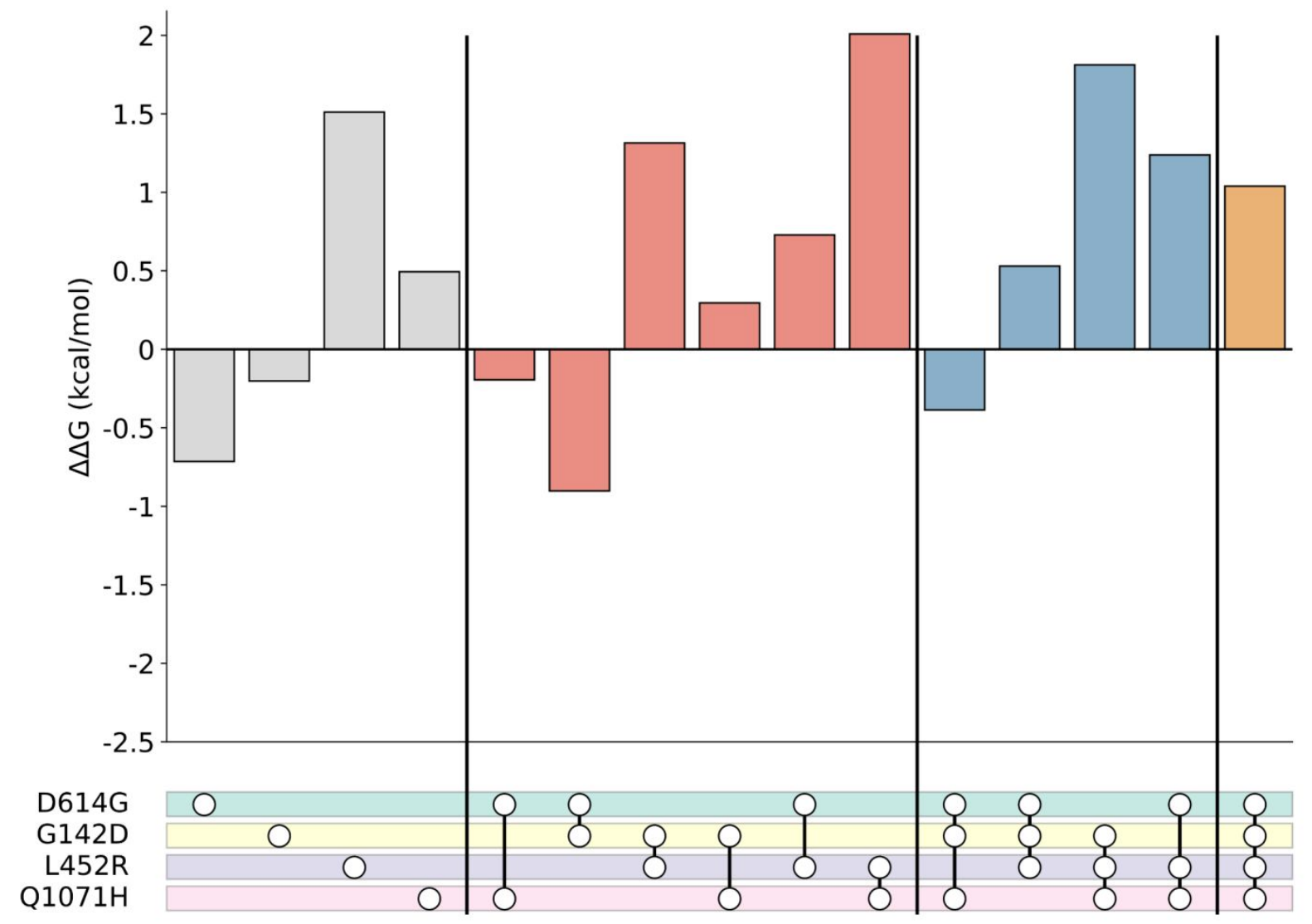

Figure S12: Upset plot for mutation combinations in SARS-CoV-2 Kappa variant.

Table S1: Table containing predicted $\Delta \Delta \mathrm{G}$ for every possible mutations in SARS-CoV-2

structure PDBID 6VXX (available as XLSX) 


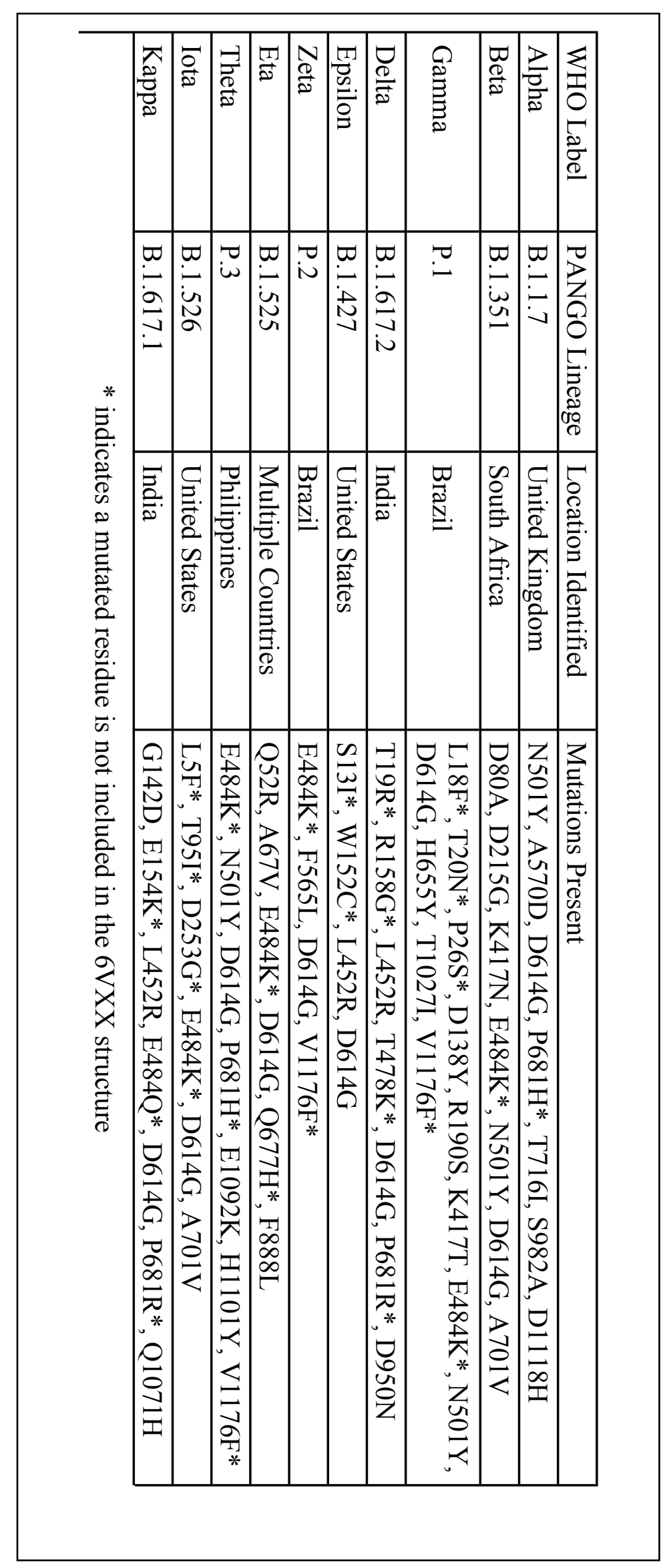


Table S2. SARS-CoV-2 Variants of Concern (Alpha, Beta, Gamma, and Delta), and Variants of Interest (Epsilon, Zeta, Eta, Theta, Iota, and Kappa) as of June 2021.

\section{References:}

(1) Walls, A. C.; Park, Y.-J.; Tortorici, M. A.; Wall, A.; McGuire, A. T.; Veesler, D. Structure, Function, and Antigenicity of the SARS-CoV-2 Spike Glycoprotein. Cell 2020, 181 (2), 281292.e6. https://doi.org/10.1016/j.cell.2020.02.058.

(2) Schymkowitz, J.; Borg, J.; Stricher, F.; Nys, R.; Rousseau, F.; Serrano, L. The FoldX Web Server: An Online Force Field. Nucleic Acids Research 2005, 33 (Web Server), W382-W388. https://doi.org/10.1093/nar/gki387.

(3) Emma B. Hodcroft. CoVariants: SARS-CoV-2 Mutations and Variants of Interest https://covariants.org/ (accessed 2021 -06 -15). 